(2)

\title{
MiR-519d represses ovarian cancer cell proliferation and enhances cisplatin-mediated cytotoxicity in vitro by targeting XIAP
}

This article was published in the following Dove Press journal:

OncoTargets and Therapy

23 April 2014

Number of times this article has been viewed

\author{
Yingxin Pang' \\ Hongluan Mao' \\ Liang Shen ${ }^{2}$ \\ Zhe Zhao' \\ Ruihan Liu' \\ Peishu Liu' \\ 'Department of Obstetrics and \\ Gynecology, Qilu Hospital of \\ Shandong University, Jinan, People's \\ Republic of China; ${ }^{2}$ Department of \\ Obstetrics and Gynecology, \\ Provincial Hospital Affiliated \\ with Shandong University, Jinan, \\ People's Republic of China
}

Background: MicroRNAs (miRNAs) are small, noncoding RNAs that are believed to play fundamental roles in tumorigenesis and tumor development at the posttranscriptional level, as negative regulators of gene expression. This study was designed to evaluate the expression and anticancer effect of miR-519d in ovarian cancer.

Methods: The expression levels of miR-519d in ovarian cancer cells and tissues were detected by TaqMan quantitative reverse transcriptase-polymerase chain reaction (TaqMan qRT-PCR; Life Technologies, Carlsbad, CA, USA). The effects of miR-519d on ovarian cancer cell proliferation and cisplatin chemosensitivity were analyzed by 3-(4,5-dimethylthiazol-2-yl)-2, 5-diphenyltetrazolium bromide, flow cytometry, and Western blotting assay. A luciferase reporter assay was performed to validate the miR-519d binding sites on the $3^{\prime}$ untranslated region of X-linked inhibitor of apoptosis protein (XIAP). The expression levels of XIAP mRNA and protein were examined by quantitative real-time polymerase chain reaction (qRT-PCR) and Western blotting assay, respectively.

Results: miR-519d was significantly downregulated in human ovarian cancer cell lines and tissues. Overexpression of miR-519d in ovarian cancer cells decreased cell proliferation and sensitized ovarian cancer cells to cisplatin-induced cell death accompanied by increased activation of caspase 3 and cleavage of poly(adenosine diphosphate [ADP]-ribose) polymerase 1 . Bioinformatics analysis indicated that XIAP was a putative target of miR-519d. Overexpression of miR-519d decreased XIAP expression at both the protein and mRNA levels. In contrast, inhibition of miR-519d increased XIAP expression. Luciferase reporter assay confirmed XIAP as a direct target of miR-519d. XIAP mRNA and protein expression levels were inversely correlated with miR-519d expression in ovarian cancer cell lines and tissues.

Conclusion: These findings indicate that miR-519d suppresses cell proliferation and sensitizes ovarian cancer cells to cisplatin-induced cell death by targeting the XIAP transcript, suggesting that miR-519d plays a tumor-suppressive role in human ovarian cancer and highlighting the therapeutic potential of miR-519d in ovarian cancer treatment.

Keywords: microRNA, ovarian cancer, cisplatin, X-linked inhibitor of apoptosis protein, apoptosis

\section{Introduction}

Ovarian cancer is the most lethal female gynecologic malignancy, accounting for $\sim 15,500$ deaths annually in the United States, and the 5-year relative survival rates keep lingering on a lower level in the long-term. ${ }^{1}$ The development of chemoresistance to clinical first-line chemotherapeutic agents (eg, cisplatin and paclitaxel) results in low cure rates and severely limits successful treatment. ${ }^{2}$ However, the development of effective chemotherapy for advanced ovarian cancer remains rather slow.
Correspondence: Peishu Liu

Gynecology, Qilu Hospital of

Shandong University, No 107 Wen

Hua Xi Road, Jinan, Shandong 250012 ,

People's Republic of China

Tel +86 53I 82169571

Fax +8653196927544

Email peishuliu@।26.com 
Therefore, searching for new alternatively effective regimens or chemicals that can improve chemotherapy response is urgently needed.

In recent years, a new class of regulatory molecules, called microRNAs (miRNAs), have been shown to be critical players in cancer development and progression. ${ }^{3-5}$ miRNAs are an abundant class of small ( $\sim 22$ nucleotides long) endogenous noncoding RNAs that regulate gene expression at the posttranscriptional level and induce translational repression, mRNA cleavage, or destabilization by base-pairing to the 3 '-untranslated region ( 3 '-UTR) of the target mRNAs in animals and plants. ${ }^{6,7}$ Most recently, miRNA has received attention for its role in regulating cancer cell response to chemotherapy drugs in ovarian cancer cells. Several miRNAs are abnormally expressed in chemoresistant compared with chemosensitive ovarian cancer cells. For example, miR-130a was downregulated in a paclitaxel-resistant A2780/paclitaxel (TAX) cell line and targeted Microphage colony stimulating factor (M-CSF). ${ }^{8}$ Aberrant expression of miR-130a has also been reported to be associated with cisplatin resistance in a cisplatin-resistant SKOV3/cisplatin (CIS) cell line, which abnormally expresses high levels of MDR1/P-glycoprotein. ${ }^{9}$ Moreover, miR-214 induced cell survival and caused cisplatin resistance by targeting the 3'-UTR of a common tumor suppressor gene, phosphatase and tension homolog (PTEN). ${ }^{10}$ In another recent study aimed at identifying chemosensitivityrelated miRNAs in vitro, 27 miRNAs were shown to be associated with one or more clinical chemotherapeutic agent in ovarian cancer cell lines. ${ }^{11}$ Gene pathway analysis on predicted target messenger RNAs (mRNAs) of these miRNAs involved cancer cell cytotoxicity, mitosis, p53 signaling pathway, and tumor cell proliferation and invasion. Analysis of the deregulated miRNAs and their clinicopathologic significance in human ovarian cancer may be useful in developing novel strategies of targeted therapies.

MiR-519d is a member of the largest human miRNA cluster (C19MC), which originates from chromosome $19 .^{12}$ Recent studies of miR-519d-mediated gene regulation have shown involvement in human hepatocellular carcinoma (HCC), pathologic pregnancy, and obesity. ${ }^{13-16}$ Several reports also showed that miR-519d displays very low expression in most human tissues and that upregulation of miR-519d was involved in HCC by p53 and DNA hypomethylation. ${ }^{12,13}$ However, another study detected lower expression levels of miR-519d in HCC tissues compared with in the matched normal tissues and identified miR-519d as a tumor suppressor molecular by targeting MKi67 and inhibiting cell growth in HCC cells. ${ }^{15}$ Recently, miR-519d was noted to be downregulated in advanced ovarian cancer tissues. ${ }^{17}$ However, little is known about the role of miR-519d and its target mRNAs in ovarian cancer. In this study, we sought to determine whether miR-519d is aberrantly expressed in ovarian cancer and to identify potential targets of miR-519d. Here, we show that X-linked inhibitor of apoptosis protein (XIAP) protein expression exhibited an inverse correlation with miR519d expression levels that is significantly downregulated in ovarian cancer cells and tissues. MiR-519d represses cell proliferation and facilitates ovarian cancer cells to cisplatininduced cell death by down-regulation of XIAP protein, which may provide a potential target for gene therapy.

\section{Materials and methods Cell lines and drug treatment}

Human ovarian cancer cell lines (A2780, OVCAR3, and SKOV3) were obtained from the Cell Bank of the Chinese Academy of Sciences in Shanghai, People's Republic of China. A2780 and OVCAR3 cells were cultured in Roswell Park Memorial Institute 1640 medium (Gibco, Auckland, New Zealand). SKOV3 cells were cultured in McCoy's 5A medium (Sigma-Aldrich, St Louis, MO, USA). All cells were cultured at $37^{\circ} \mathrm{C}$ with $5 \% \mathrm{CO}_{2}$ in media supplemented with $10 \%$ fetal bovine serum (TBD, Tianjin, People's Republic of China) and $100 \mathrm{U} / \mathrm{mL}$ penicillin and $100 \mu \mathrm{g} / \mathrm{mL}$ streptomycin. Cisplatin was purchased from Qilu Pharmaceutical (Jinan, People's Republic of China). Until reaching approximately $70 \%-80 \%$ confluence, cells were treated with chemicals, as indicated. Control cells were subjected to vehicle treatment. Media with or without chemicals were changed daily.

\section{Patient samples}

All tissue samples were collected at the Qilu Hospital of Shandong University. Ovarian cancer tissues were obtained from patients diagnosed as having primary stage III or IV ovarian cancer, with an average age of 62 years. None of the patients had received chemotherapy before surgery. All samples were pathologically proven to be serous ovarian cancer (grade 2 or 3). Informed consent was obtained from each patient. Samples were immediately frozen by liquid nitrogen for protein or RNA extraction. This study was approved by the ethics committee at Shandong University.

\section{MiRNA and siRNA transfection}

Cells were plated in 96- or 6-well plates 1 day before transient transfection. Transfection was performed using Lipofectamine 2000 reagent (Invitrogen Life Technologies, Carlsbad, CA, USA) in OPTI-MEM media (Gibco) and miRNA mimic (100 
$\mathrm{nm})$, negative control $(100 \mathrm{~nm})$, miRNA inhibitor $(200 \mathrm{~nm})$, inhibitor control (200 nm) (GenePharma Co, Ltd, Shanghai, People's Republic of China), or XIAP siRNA (Invitrogen Life Technologies). Analyses were performed 48-72 hours after transfection. The XIAP siRNA sequences were as follows: 5'-UUCAACUUUGUACUGACCAUUCUGG-3', 5'-CCAG AAUGGUCAGUACAAAGUUGAA-3'.

\section{Cell viability and cell death assay}

Cells were seeded in 96-well culture plates for cell viability assay, and cells were plated in 6-well plates for cell death assay. Then, cells were transfected with miRNA mimic or negative control or XIAP siRNA for 48 or 72 hours. In some experiments, after 48 hours of transfection, cells were exposed to indicated concentrations of cisplatin for another 12 hours. Cell viability was determined via 3-(4,5-dimethylthiazol2-yl)-2,5-diphenyl-2H-tetrazoliumbromide (MTT; SigmaAldrich) assay on a plate reader (Bio-Rad, Hercules, CA, USA). Cell death was measured by Annexin V/picolinium iodide (PI) double-staining assay with flow cytometry (BD Biosciences, San Jose, CA, USA). Three replicates were used for each treatment, and the assay was repeated at least three times.

\section{Western blotting}

After transfection and/or treatment with chemicals, cells were lysed for Western blot assay, as described previously. ${ }^{18}$ Protein aliquots (50-100 $\mu \mathrm{g} /$ lane) were taken for protein separation by sodium dodecyl sulfate polyacrylamide gel electrophoresis. Primary antibodies used for immunodetection were anti-poly(adenosine diphosphate ribose [ADPribose]) polymerase-1 (PARP-1) (catalog no sc-7150), anti-glyceraldehyde 3-phosphate dehydrogenase (GAPDH) (catalog no sc-47724; Santa Cruz Biotechnology, Dallas, TX, USA), anti-caspase-3 (catalog no 9668), and anti-XIAP (catalog no 2045; Cell Signaling Technology, Danvers, MA, USA). Secondary antibodies were anti-rabbit and anti-mouse IgG peroxidase conjugate (Zhongshan Jinqiao Biotechnology Co, Ltd, Beijing, People's Republic of China). Fold changes in protein were determined on the basis of GAPDH loading control. The results of Western blotting were quantified by densitometry, using Image $\mathrm{J}$ software.

\section{Heuristic computational analysis of miRNA candidates}

The analysis of miRNA potential candidates was determined using the algorithms in TargetScan 5.2 software (Bartel Laboratory, Boston, MA, USA). ${ }^{19}$

\section{RNA extraction and quantitative real-time PCR analyses of mRNA}

Total RNAs were isolated using Trizol (Invitrogen Life Technologies), according to the manufacturer's instructions. One microgram total RNA was used for reverse transcription, using a ReverTra Ace qPCR-RT Kit (Toyobo, Osaka, Japan) in a reaction volume of $10 \mu \mathrm{L}$. qRT-PCR was performed to detect the relative level of XIAP mRNA, using the LightCycler rapid thermal cycler system 2.0 (Roche Diagnostics Ltd, Burgess Hill, United Kingdom) with SYBR Green Master Mix (Toyobo). Primers used for real-time PCR are as follows: XIAP (forward) 5'-CCGTGCGGTGCTTTAGTTGT-3' (reverse) 5'-TTCCTCGGGTATATGGTGTCTGAT-3'; GAPDH (forward) 5'-TGGTCACCAGGGCTGCTT-3' (reverse) 5'-AGCTTCCCGTTCTCAGCCTT-3'. Real-time qRT-PCR was performed with cycle parameters of $95^{\circ} \mathrm{C}$ for 5 minutes, followed by 40 cycles of $95^{\circ} \mathrm{C}$ for 15 seconds, $58^{\circ} \mathrm{C}$ for 20 seconds, and $72^{\circ} \mathrm{C}$ for 20 seconds. The relative concentrations of the PCR products were calculated using LightCycler System software. All samples were normalized to GAPDH expression, using the $2^{-\Delta \Delta \mathrm{Ct}}$ or $2^{-\Delta \mathrm{Ct}}$ method. ${ }^{20,21}$ All experiments were conducted in triplicate.

\section{Real-time RT-PCR of miRNA}

For quantification of miRNA-519d expression levels, RTPCR reactions were performed using the TaqMan microRNA reverse transcription kit (Applied Biosystems, Carlsbad, CA, USA) and the TaqMan Universal PCR Master Mix (Applied Biosystems), according to the manufacturer's protocols. All reactions were performed in triplicate, and U6 snRNA was taken as internal control. Results were analyzed using the $2^{-\Delta \Delta \mathrm{Ct}}$ or $2^{-\Delta \mathrm{Ct}}$ method.

\section{Luciferase assay}

To verify the precise target of miRNA-519d, the pmirGLO system (Promega, Madison, WI, USA) was applied. The wild-type and mutant 3 '-UTR fragments of XIAP containing the predicted miRNA-519d binding sites were cloned into the pmirGLO Dual-Luciferase miRNA Target Expression Vector. Briefly, oligonucleotides containing the predicted miRNA binding sites in XIAP 3'-UTR were synthesized by GenePharma (Shanghai, People's Republic of China) and obtained by annealing, purifying, and cloning into pmirGLO vector between the Pmel and $\mathrm{XbaI}$ restriction sites according to the manufacturer's protocol. As a control, the pmirGLOXIAP-mut plasmid was also prepared by the same method.

Synthetic oligonucleotides are 5'-AAACTAGCGGCCG CTAGTCTACATATTAAATGACACTTTATAACTAAT-3' 
(position 1228-1234, wild type), 5'-AAACTAGCGGCCG CTAGTTAAGCCTGCCTAAGTGAGATACTAAAAGT-3' (position 1228-1234, mutate type), 5'-AAACTAGCGGCC GCTAGTCTACATATTAAATGACACTTTATAACTAAT-3' (position 4925-4931, wild type), and 5'-AAACTAGCGGCCG CTAGTCTACATATATTATGACTCATATAACTAAT-3' (position 4925-4931, mutate type). In 24-well plates, SKOV3 cells were transfected with miR-519d or negative control and then cotransfected with wild-type or mutant vectors. After 48 hours of transfection, the cells were lysed for luciferase assays using the dual luciferase assay system (Promega).

\section{Statistical analysis}

All experiments were performed at least three independent times in triplicate. Results were expressed as the mean \pm standard deviation. Two-tailed Student's $t$-test was performed to assess differences between experimental groups and control group, and $P<0.05$ was considered statistically significant. All statistical analyses were performed with GraphPad Prism Version 5.01 (GraphPad software, La Jolla, CA, USA).

\section{Results}

\section{MiR-5I9d was downregulated in human ovarian cancer cells and tissues}

To investigate whether miR-519d was abnormally expressed in ovarian cancer, as Zhang et al have reported, ${ }^{17}$ we performed the TaqMan-based real-time RT-PCR analyses to examine expression levels of miR-519d in three ovarian cancer cell lines. Normal ovarian tissues were used as the reference. As shown in Figure 1A, compared with normal ovarian samples, all ovarian cancer cell lines (OVCAR3, A2780, and SKOV3) showed significantly lower expression of miR-519d $(P<0.05)$, suggesting a possible role of this miRNA involved in ovarian cancer. Next, we detected miR-519d expression in ovarian cancer tissues and normal ovarian tissues. We found that miR-519d was significantly downregulated in seven ovarian cancer tissues compared with in two normal ovarian tissues (Figure 1B).

\section{MiR-5 I9d inhibited the proliferation of ovarian cancer cells}

For forced upregulation or downregulation of miR-519d, we used chemically synthesized oligonucleotides and transfected them into ovarian cancer A2780 and SKOV3 cells. Two days after transfection, relative expression of miR-519d was detected with TaqMan qRT-PCR (Figure 2A and B). The in vitro growth ability of ovarian cancer cells was determined
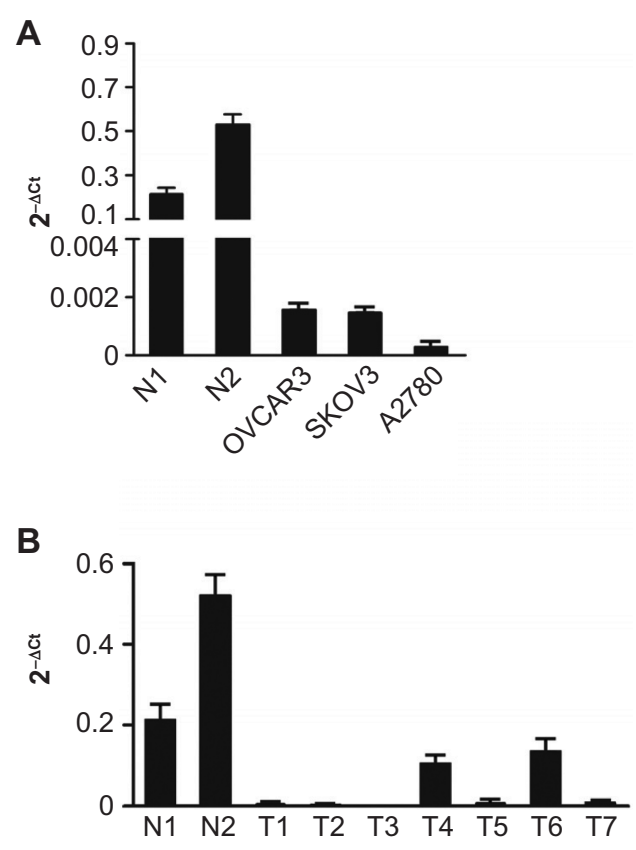

Figure I MiR-5I9d was downregulated in human ovarian cancer. (A) Relative expression of miR-519d in normal ovarian tissues and a series of ovarian cancer cell lines. (B) Expression of miR-519d in normal ovarian tissues and cancer tissues. Notes: Relative expression of miR-519d was detected by TaqMan quantitative reverse transcriptase-polymerase chain reaction microRNA assays and normalized to U6 small nuclear RNA. The relative amount of miR-519d was described using the $2^{-\Delta \Delta C t}$ method. $P<0.05$ compared with the control group, as indicated. Data are displayed in means \pm standard deviation of at least three independent experiments of each group.

Abbreviations: N, normal tissues; T, cancer tissues; MiR-519d, microRNA-519d.

by MTT assay. Inhibition rates of miR-519d transfectants were about $15.50 \% \pm 3.62 \%$ and $29.70 \% \pm 8.31 \%$ in $\mathrm{A} 2780$ cells at 48 and 72 hours, respectively $(P<0.05)$ (Figure 2C). However, there was no statistical difference in SKOV3 cells $(P>0.05)$ (Figure 2D).

\section{MiR-5I9d sensitized ovarian cancer cells to cisplatin-induced cell death}

To determine the hypothesis that miR-519d functions as a tumor suppressor in ovarian cancer cells, we next examined whether miR-519d is involved in cisplatin-induced cell death in ovarian cancer cells. As shown in Figure 3A and $\mathrm{B}$, overexpression of miR-519d promoted cisplatininduced proliferation inhibition in ovarian cancer A2780 and SKOV3 cells. Similarly, the addition of miR-519d remarkably facilitated cisplatin-induced apoptosis (Figure $3 \mathrm{C}$ and $\mathrm{D}$ ). The percentage of late apoptotic cells (Annexin V-fluoresceine isothiocyanate (FITC)+/PI+) increased significantly in A2780 cells transfected with miR-519d compared with control cells, whereas both early (Annexin V-FITC+/PI-) and late (Annexin V-FITC+/PI+) apoptosis and necrotic rates (only PI-positive) were more evident in 
A

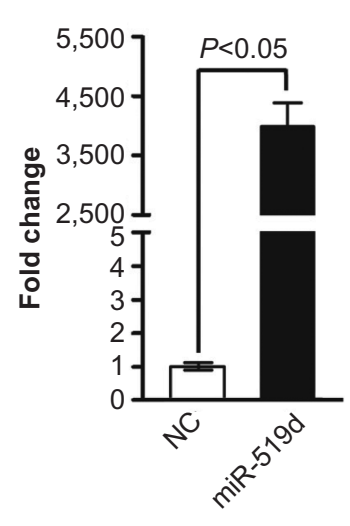

C

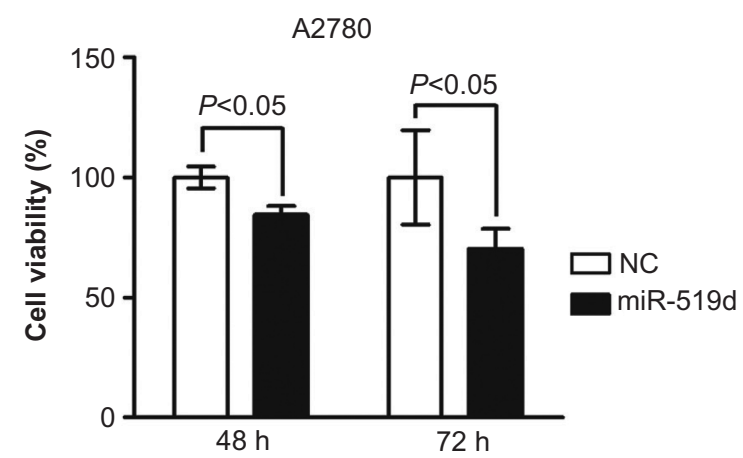

B

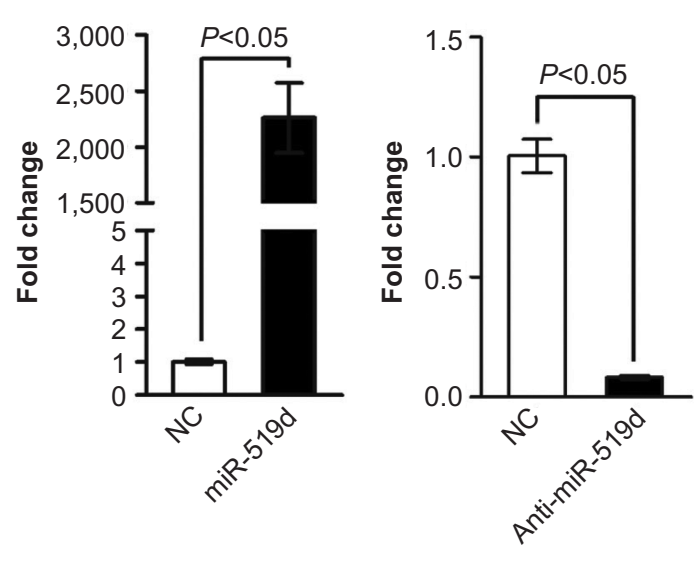

D

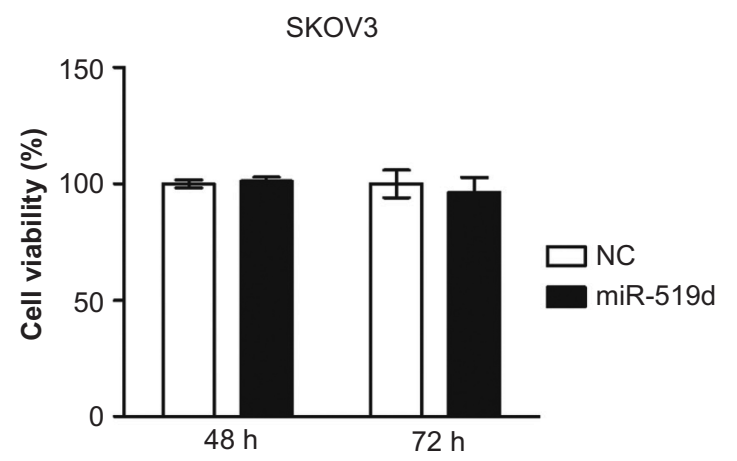

Figure 2 Effect of miR-519d on cell proliferation in human ovarian cancer cells. Relative expression of miR-519d in A2780 (A) and SKOV3 (B) cells after transfection with the mimic or inhibitor of miR-519d $(P<0.05)$. The growth of A2780 (C) and SKOV3 (D) cells transfected with the mimics was determined by 3-(4,5-dimethylthiazol-2-yl)-2, 5-diphenyltetrazolium bromide assay at 48 and 72 hours $(P<0.05)$.

Notes: $P<0.05$ compared with control group, as indicated. The results are representative of three different experiments. Data are expressed as mean \pm standard deviation.

Abbreviations: h, hours; NC, negative control; MiR-519d, microRNA-519d.

miR-519d-treated SKOV3 cells compared with a negative control group. Furthermore, we also validated that overexpression of miR-519d markedly facilitated cisplatin-induced cleavage of caspase 3 and PARP-1, a well-known product of active caspase 3 (Figure 3E and F). These findings suggested a proapoptotic effect of miR-519d on ovarian cancer cells.

\section{XIAP is a direct target of miR-5I9d}

To identify direct $m i R-519 d$ target genes, TargetScan was used. As shown in Figure 4A, the 3'-UTR of XIAP is predicted to contain two putative miR-519d-binding sites. To validate regulation of XIAP by miR-519d, we first tested whether overexpression of miR-519d in ovarian cancer A2780 and SKOV3 cells would have an effect on XIAP protein level. As shown in Figure 4B and C, overexpression of miR-519d significantly reduced XIAP protein level after 48 hours of transfection with miR-519d mimic. In contrast, inhibition of miR-519d by miRNA inhibitor increased XIAP protein level in A2780 and SKOV3 cells. These findings were also validated at the mRNA level on miR-519d overexpression or inhibition (Figure 4D), confirming mRNA degradation mechanisms for miR-519d targeting of XIAP. To further validate XIAP as a direct target of miR-519d, we next performed a luciferase reporter assay. Relative luciferase activity was significantly reduced for both candidate sites of XIAP 3'-UTR (Figure 4E and F), indicating that XIAP is a potential direct target of miR-519d. Mutations in the predicted miR-519d target sites abrogated the inhibition by miR-519d mimic, confirming the functionality of these target sites. The results of the luciferase activity assay confirmed that XIAP is indeed a direct target of miR-519d.

\section{Downregulation of XIAP inhibits the growth of ovarian cancer cells and promotes cisplatin-mediated cytotoxicity}

To further validate the idea that miR-519d-mediated effects in ovarian cancer cells resulted from targeting XIAP, siRNA strategy was used to knockdown XIAP. XIAP siRNA effectively reduced XIAP protein levels (Figure 5A). 
A

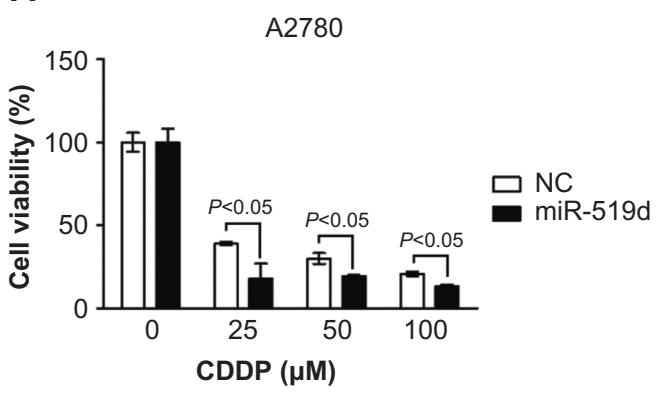

B

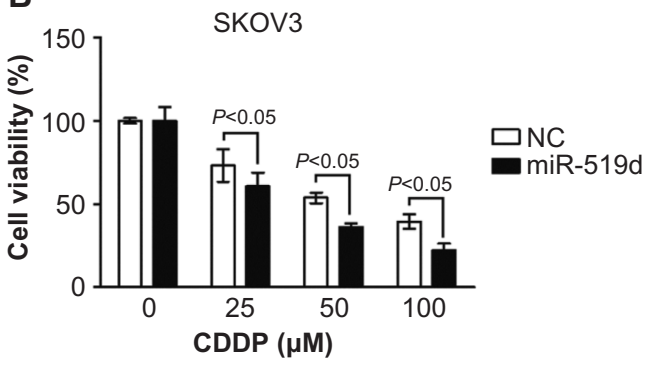

C

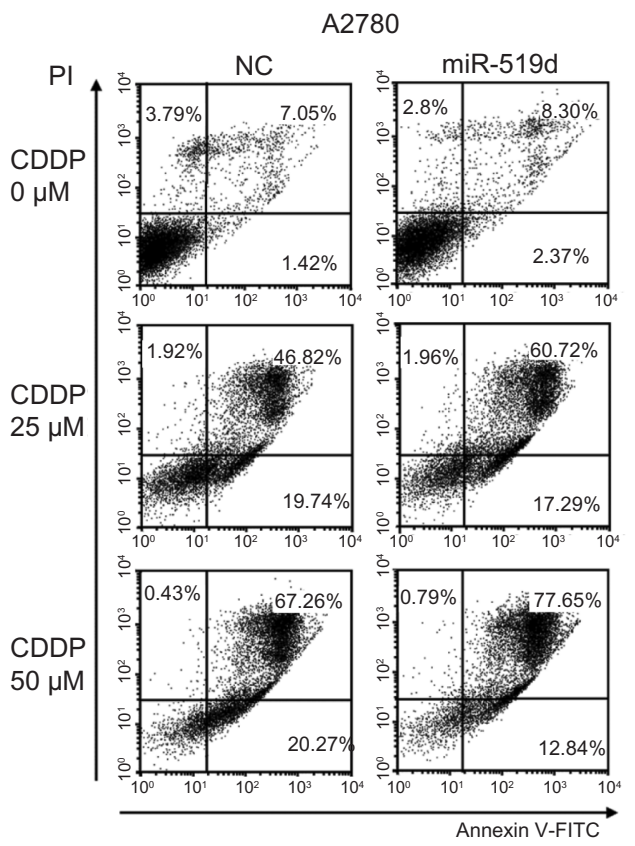

D

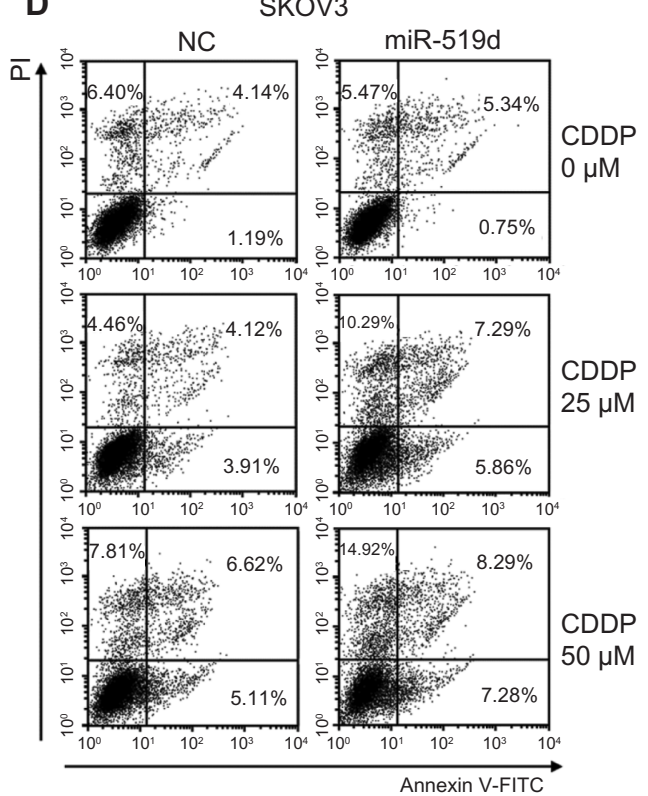

E

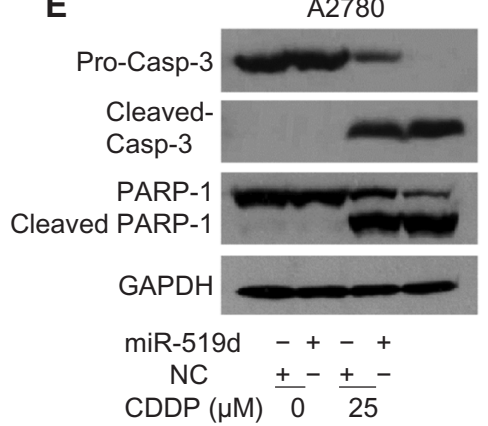

F

SKOV3

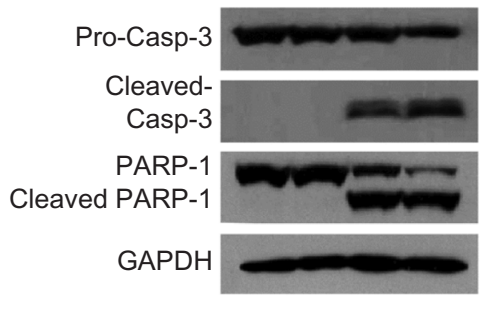

$\operatorname{miR}-519 \mathrm{~d}-+++$

$\mathrm{NC}(\mu \mathrm{M}) \stackrel{+}{0}-\frac{+}{50}^{-}$

Figure 3 miR-519d sensitizes ovarian cancer cells to cisplatin-induced cell death. (A and B) Changes in cell viability in treatment with cisplatin in the presence or absence of miR-519d were detected by MTT assay $(P<0.05)$. (C and $\mathbf{D})$ Effect of the alteration of miR-519d expression on cisplatin-treated ovarian cancer cell death was determined by flow cytometric analysis in A2780 and SKOV3 cells. (E and F) Changes in expressions of apoptosis-related proteins were determined by Western blotting analysis in A2780 and SKOV3 cells.

Notes: A2780 cells or SKOV3 cells were transfected with miR-519d (100 nm) or negative control $(100 \mathrm{~nm})$ for 48 hours before the challenge with indicated concentrations of cisplatin for further 12 hours. $P<0.05$ compared with control group, as indicated. The results are representative of three different experiments. Data are expressed as mean \pm standard deviation.

Abbreviations: CDDP, cisplatin; Casp-3, caspase 3; PARP-I, poly(ADP-ribose) polymerase-I; MTT, 3-(4,5-dimethylthiazol-2-yl)-2,5-diphenyl-2H-tetrazoliumbromide; ADP, adenosine diphosphate; MiR-519d, microRNA-519d; GAPDH, glyceraldehyde-3-phosphate dehydrogenase; FITC, fluoresceine isothiocyanate; NC, negative control; PI, picolinium iodide; ADP, adenosine diphosphate.

The transient transfection of XIAP siRNA into A2780 cells indicated that knockdown of XIAP could suppress cell proliferation in 48 and 72 hours, as determined by MTT assay $(P<0.05)$; however, downregulation of XIAP could not inhibit cell growth in SKOV3 cells $(P>0.05)$ (Figure 5B).
XIAP siRNA also promoted cisplatin-induced proliferation inhibition and facilitated cleavage of apoptosis-related proteins, caspase 3 and PARP-1, in both ovarian cancer cell lines (Figure 5C and D). These results are consistent with the finding that miR-519d can inhibit ovarian cancer 


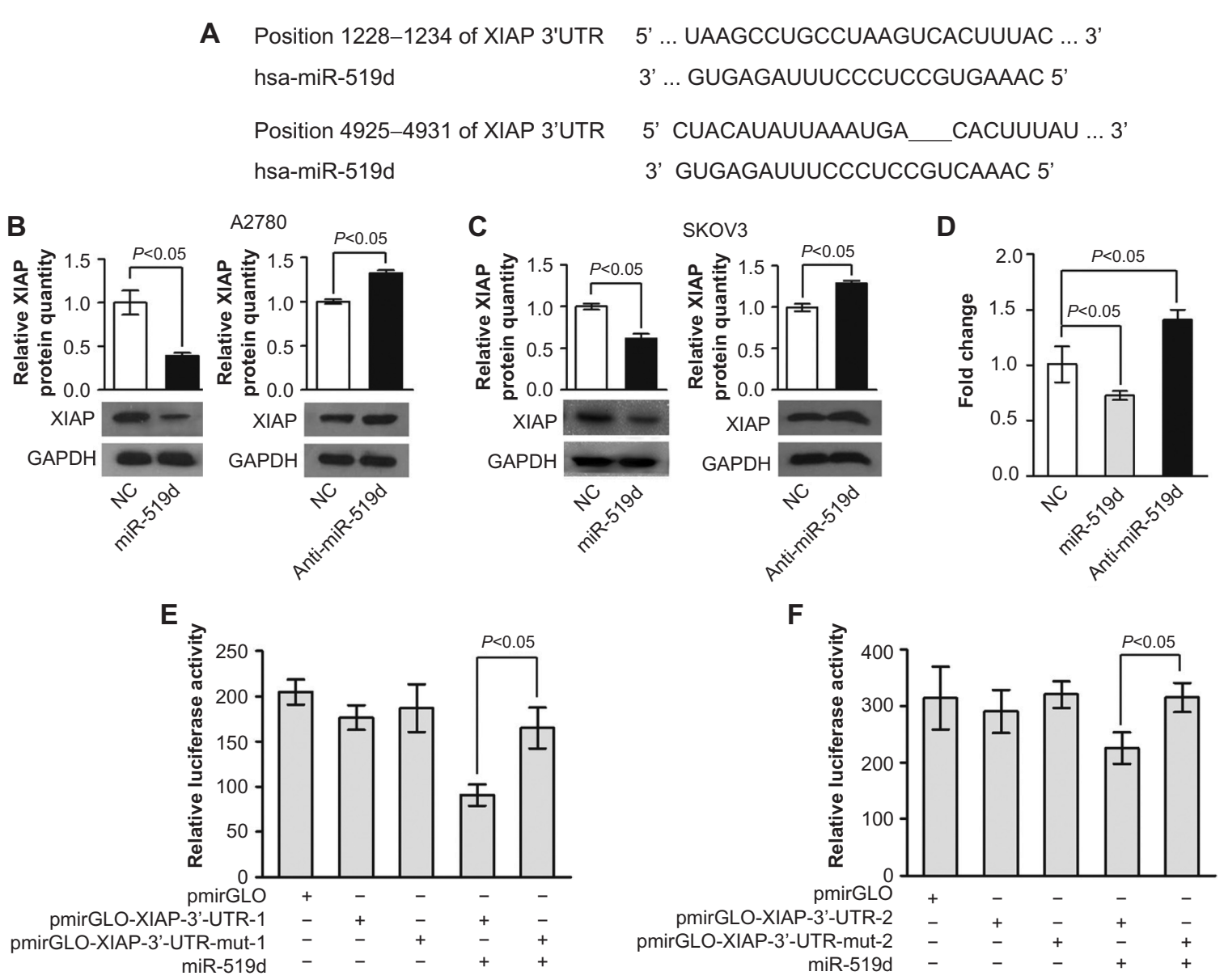

Figure 4 miR-519d inhibits XIAP expression. (A) Schematic graph of the putative two binding sites of miR-519d in the XIAP $3^{\prime}$ untranslated region predicted by TargetScan (Bartel Laboratory, Boston, MA, USA). Detection of XIAP protein (B and C) and messenger RNA (D) expression in A2780 and/or SKOV3 cells by Western blotting and quantitative reverse transcriptase-polymerase chain reaction analyses following transfection with miR-519d mimic or inhibitor. (E and $\mathbf{F}$ ) Luciferase reporter assays indicate direct interactions between miR-519d and XIAP.

Notes: After transfection with miR-519d mimic $(100 \mathrm{~nm})$ or inhibitor $(200 \mathrm{~nm})$ for 48 hours, cells were harvested for protein or RNA extraction. After 48 hours of cotransfection with miR-5I9d mimic and indicated vectors containing wild-type or mutant $3^{\prime}$ untranslated region of XIAP, the cells were lysed for luciferase assays, using the dual luciferase assay system. The results are representative of three different experiments. Data are expressed as mean \pm standard deviation.

Abbreviations: XIAP, X-linked inhibitor of apoptosis protein; XIAP-3'-UTR-I, pmirGLO-XIAP-3'-UTR-I 228-I234; XIAP-3'-UTR-2, PmirGLO-XIAP-3'-UTR-4925-493I; miR-5I9d, microRNA-519d; UTR, untranslated region; GAPDH, glyceraldehyde-3-phosphate dehydrogenase; hsa, homo sapiens; NC, negative control.

cell growth in vitro and sensitize cisplatin-mediated cytotoxicity.

\section{Quantitative analysis of XIAP and miR-5I9d expression in human ovarian cancer cells and tissues}

To determine the relationship between miR-519d and XIAP, we assayed the relative abundance of miR-519d in ovarian cancer cell lines (A2780, SKOV3, and OVCAR3). MiR-519d expression was highest in OVCAR3 cells and lowest in A2780 cells (Figure 6A). In contrast, using RTPCR, relative expression of XIAP mRNA exhibited the highest expression in A2780 cells and was undetectable in OVCAR3 cells (Figure 6B). The protein levels of XIAP in the three ovarian cancer cells were consistent with XIAP mRNA expression levels (Figure 6C). We also detected XIAP protein expression in ovarian normal and cancer tissues.
XIAP protein expression exhibited an inverse correlation with miR-519d expression levels (Figures 1B and 6D). In general, miR-519d exhibited negative correlation with XIAP.

\section{Discussion}

Recurrence of ovarian cancer is mainly caused by drug resistance, which generally exhibits a multifactorial process. In advanced ovarian cancer, combination cisplatin with paclitaxel is considered the first line of chemotherapy. However, at least one-fifth of patients are either unresponsive in the first cycle or progress within the first year of treatment. ${ }^{22}$ Within the progression of ovarian cancer, the remaining patients become resistant to chemotherapy and relapse with poor outcome. Therefore, it is necessary to identify specific molecules involved in drug resistance, as well as new therapeutic approaches. Accumulating evidence shows that miRNAs may play an important role in such processes. In fact, miRNAs 
A

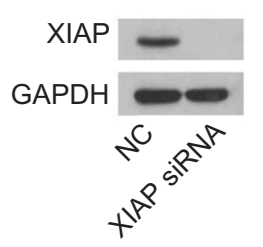

B

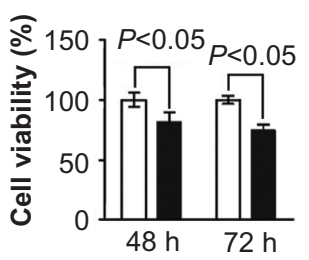

SKOV3

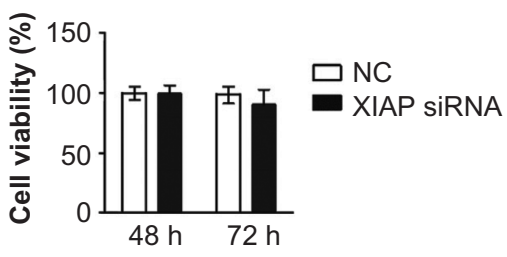

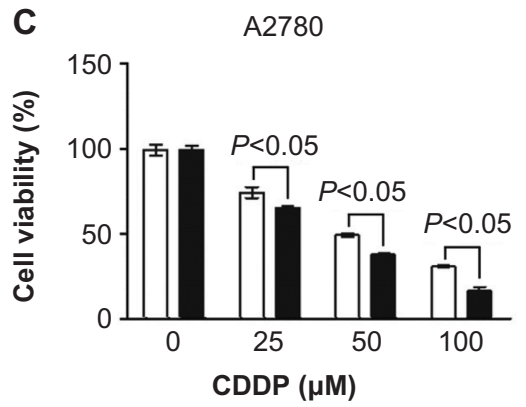

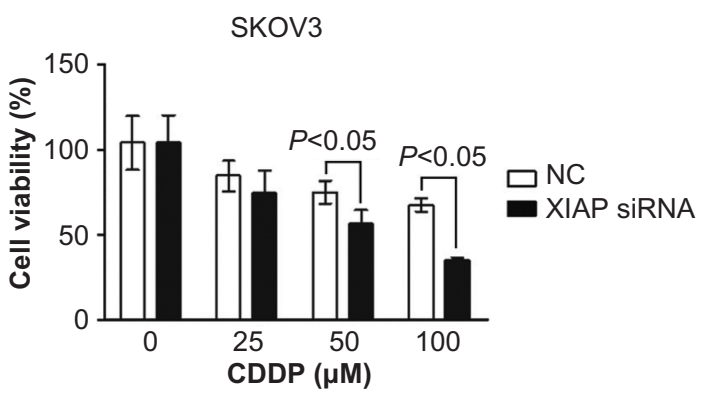

D
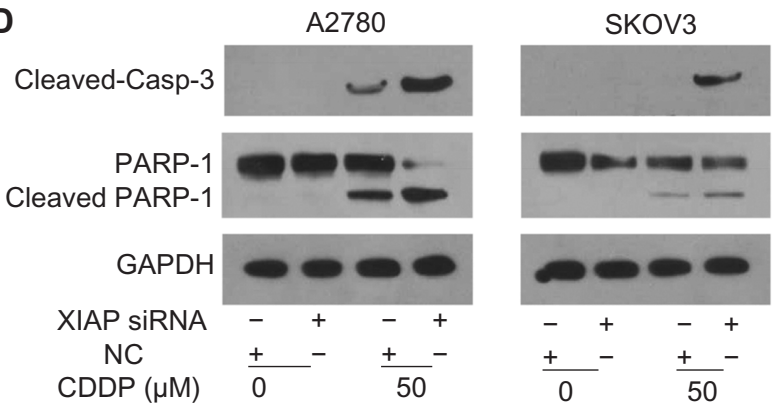

Figure 5 Downregulation of XIAP inhibits the growth of ovarian cancer cells and promotes cisplatin-mediated cytotoxicity. (A) XIAP protein expression in A2780 cells transfected with XIAP siRNA for 48 hours was determined by Western blotting analysis. (B) The growth of A2780 and SKOV3 cells transfected with XIAP siRNA was determined by MTT assay at 48 and 72 hours. (C) Changes in cell viability in treatment with cisplatin in the presence or absence of XIAP siRNA were detected by MTT assay $(P<0.05)$. (D) Changes in expressions of apoptosis-related proteins were determined by Western blotting analysis in A2780 and SKOV3 cells.

Notes: A2780 cells or SKOV3 cells were transfected with XIAP siRNA for 48 hours before the challenge with indicated concentrations of cisplatin for a further 12 hours. $P<0.05$ compared with control group, as indicated. The results are representative of three different experiments. Data are expressed as mean \pm standard deviation. Abbreviations: XIAP, X-linked inhibitor of apoptosis protein; siRNA, small interfering RNA; CDDP, cisplatin; Casp-3, caspase 3; PARP-I, poly(ADP-ribose) polymerase I; MTT, 3-(4,5-dimethylthiazol-2-yl)-2,5-diphenyl-2H-tetrazoliumbromide; GAPDH, glyceraldehyde-3-phosphate dehydrogenase; ADP, adenosine diphosphate; NC, negative control.

are involved in many biological processes, including cell growth and death, stress resistance, and fat metabolism, as well as cancer and inflammation through the fine regulation of gene expression. ${ }^{3,23,24}$ Current studies provide evidence that intervention of miRNA dysregulation is a potential novel therapeutic approach for ovarian cancer. ${ }^{25-27}$

A recent study reported that miR-519d was downregulated in advanced ovarian cancer tissues. ${ }^{17}$ Using a TaqMan quantitative RT-PCR, we found significantly lower expression of miR-519d in ovarian cancer cell lines and tissues compared with normal ovarian tissues. A previous study also showed that miR-519d has a lower expression level in HCC tissues and was inversely correlated with expression levels of MKi67, which was confirmed as a direct target gene of miR-519d, using an enhanced green fluorescent protein (EGFP) reporter system. ${ }^{15}$ However, little is known about how miR-519d is regulated. Potential mechanisms of the aberrantly expressed miRNAs show involvement in epigenetic modification, genomic rearrangements, singlenucleotide polymorphism in the primary-microRNA (primiRNA) sequence, and dysregulation of key enzymes in the biosynthesis of miRNA; for example, the Dicer protein. ${ }^{6}$ MiR-519d is mapped to $19 \mathrm{q} 13$ and belongs to the C19MC cluster, the expression of which is reported to be highly influenced by the methylation state of an upstream $\mathrm{CpG}$-rich region. ${ }^{28}$ Additional study about the mechanism by which miR-519d is downregulated in ovarian cancer is needed.

In combination with the cell growth inhibition role of miR-519d in this study, we postulated miR-519d as a tumor suppressor in ovarian cancer. Consistent with observations, our results indicate that miR-519d facilitated cisplatin-induced cell death, probably by the mechanism of 
A

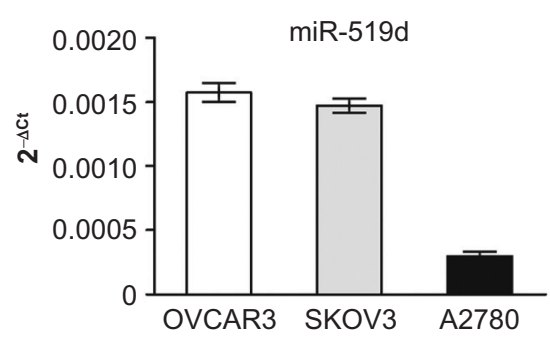

B

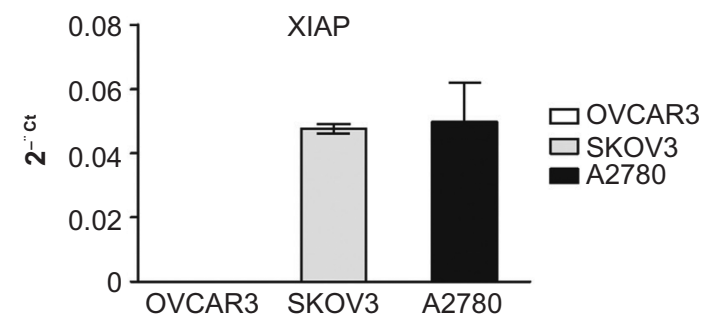

C

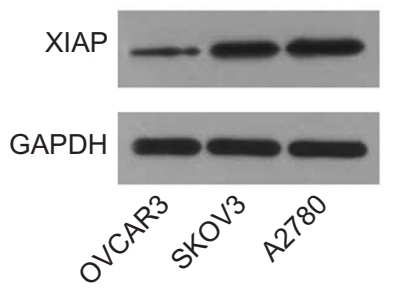

D

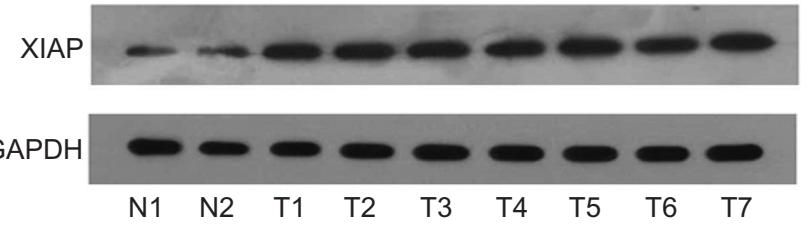

$\begin{array}{lllllllll}\mathrm{N} 1 & \mathrm{~N} 2 & \mathrm{~T} 1 & \mathrm{~T} 2 & \mathrm{~T} 3 & \mathrm{~T} 4 & \mathrm{~T} 5 & \mathrm{~T} 6 & \text { T7 }\end{array}$
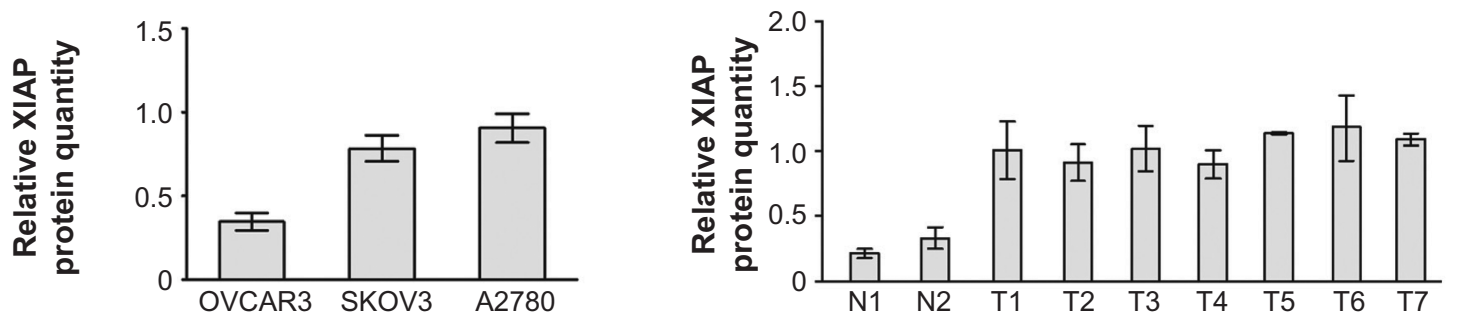

Figure 6 Quantitative analysis of miR-519d and XIAP expression in human ovarian cancer. Relative expression of miR-5I9d (A) and XIAP protein (B) and XIAP messenger RNA (C) in three ovarian cancer cell lines. (D) Relative expression of XIAP protein in normal ovarian tissues and ovarian cancer tissues.

Notes: TaqMan quantitative reverse transcriptase-polymerase chain reaction assay was performed to test the expression of miR-5I9d, and data were shown as $2^{-\Delta C t}$ method. XIAP protein and messenger RNA expressions were examined by Western blotting and reverse-transcriptase polymerase chain reaction analysis, respectively. The results are representative of three different experiments. Data are expressed as mean \pm standard deviation.

Abbreviations: XIAP, X-linked inhibitor of apoptosis protein; N, normal tissues; T, cancer tissues; miR-5I9d, microRNA-5I9d; GAPDH, glyceraldehyde-3-phosphate dehydrogenase.

downregulation of XIAP, a direct target gene predicted by computational analysis and luciferase reporter assay.

XIAP, a member of the inhibitor of apoptosis protein family, is often considered a potent endogenous inhibitor of apoptosis in ovarian cancer cells when challenged with clinical chemotherapeutic drugs such as cisplatin. ${ }^{29,30}$ Down-regulation of XIAP either by adenoviral XIAP antisense expression or specific shRNA triggering RNA interference to block XIAP gene expression induced apoptosis and enhanced cisplatinmediated cytotoxicity in cisplatin-resistant ovarian cancer cell lines, C13 and A2780/cp70, respectively. ${ }^{31,32}$ XIAP protein has three baculovirus inhibitor of apoptosis protein repeat domains that are required for caspase-inhibitive activity and a really interesting new gene (RING) zinc finger domain that is involved in protein-protein interactions and auto-ubiquitination. It has recently been determined that miRNAs can directly regulate XIAP expression. MiR-23a played an important role in ischemic sexual dimorphism through directly binding the 3'-UTR of XIAP, and suppression of miR-23a increased
XIAP mRNA level in vitro. ${ }^{33}$ Expression of XIAP can also be modulated by miR-24, the low basal expression of which resulted from genomic DNA loss at the miR-24 gene locus. ${ }^{34}$ the MiR-24 target site in XIAP $3^{\prime}$-UTR was confirmed by the luciferase reporter assay system. Overexpression of miR-24 significantly abrogated apoptosis resistance through decreasing XIAP expression in cancer cells. A recent report has also found that miR-34a*, the passenger strand, attenuated XIAP expression via targeting the XIAP 3 '-UTR in rheumatoid arthritis synovial fibroblast cells, whereas miR-34a, the corresponding mature strand, appeared to be nonfunctional. ${ }^{35}$ Our study demonstrated that XIAP is a candidate target of miR-519d in ovarian cancer. In aggregate, these findings demonstrate that miRNAs play important roles in the direct modulation of pathways involved in cell apoptosis and chemotherapeutic resistance.

In addition, we demonstrate here that miR-519d was inversely correlated with XIAP levels, which also supports our view. However, further efforts should be made to collect 
more samples and to further confirm the relationship between miR-519d and XIAP. In conclusion, miR-519d was expressed at lower levels in ovarian cancer cells and tissues, and XIAP was confirmed to be a candidate target of miR-519d. Our study is consistent with a positive role of miR-519d in sensitizing ovarian cancer cells to cisplatin. Therefore, miR-519d could be a candidate target for treatment of ovarian cancer.

\section{Disclosure}

The authors report no conflicts of interest in this work.

\section{References}

1. Siegel R, Naishadham D, Jemal A. Cancer statistics, 2012. CA Cancer J Clin. 2012;62(1):10-29.

2. Chobanian N, Dietrich CS 3rd. Ovarian cancer. Surg Clin North Am. 2008;88(2):285-299.

3. Croce CM. Causes and consequences of microRNA dysregulation in cancer. Nat Rev Genet. 2009;10(10):704-714.

4. Iorio MV, Croce CM. MicroRNA dysregulation in cancer: diagnostics, monitoring and therapeutics. A comprehensive review. EMBO Mol Med. 2012;4(3):143-159.

5. Catto JW, Alcaraz A, Bjartell AS, et al. MicroRNA in prostate, bladder, and kidney cancer: a systematic review. Eur Urol. 2011;59(5): 671-681.

6. Bartel DP. MicroRNAs: genomics, biogenesis, mechanism, and function. Cell. 2004;116(2):281-297.

7. Sontheimer EJ, Carthew RW. Silence from within: endogenous siRNAs and miRNAs. Cell. 2005;122(1):9-12.

8. Sorrentino A, Liu CG, Addario A, Peschle C, Scambia G, Ferlini C. Role of microRNAs in drug-resistant ovarian cancer cells. Gynecol Oncol. 2008;111(3):478-486.

9. Yang L, Li N, Wang H, Jia X, Wang X, Luo J. Altered microRNA expression in cisplatin-resistant ovarian cancer cells and upregulation of miR-130a associated with MDR1/P-glycoprotein-mediated drug resistance. Oncol Rep. 2012;28(2):592-600.

10. Yang H, Kong W, He L, et al. MicroRNA expression profiling in human ovarian cancer: miR-214 induces cell survival and cisplatin resistance by targeting PTEN. Cancer Res. 2008;68(2):425-433.

11. Boren T, Xiong Y, Hakam A, et al. MicroRNAs and their target messenger RNAs associated with ovarian cancer response to chemotherapy. Gynecol Oncol. 2009;113(2):249-255.

12. Bentwich I, Avniel A, Karov Y, et al. Identification of hundreds of conserved and nonconserved human microRNAs. Nat Genet. 2005;37(7):766-770.

13. Fornari F, Milazzo M, Chieco P, et al. In hepatocellular carcinoma miR-519d is up-regulated by 533 and DNA hypomethylation and targets CDKN1A/p21, PTEN, AKT3 and TIMP2. J Pathol. 2012;227(3): 275-285.

14. Martinelli R, Nardelli C, Pilone V, et al. miR-519d overexpression is associated with human obesity. Obesity (Silver Spring). 2010;18(11): 2170-2176.

15. Hou YY, Cao WW, Li L, et al. MicroRNA-519d targets MKi67 and suppresses cell growth in the hepatocellular carcinoma cell line QGY-7703. Cancer Lett. 2011;307(2):182-190.
16. Higashijima A, Miura K, Mishima H, et al. Characterization of placentaspecific microRNAs in fetal growth restriction pregnancy. Prenat Diagn. 2013;33(3):214-222.

17. Zhang L, Volinia S, Bonome T, et al. Genomic and epigenetic alterations deregulate microRNA expression in human epithelial ovarian cancer. Proc Natl Acad Sci U S A. 2008;105(19):7004-7009.

18. XiG, Hu X, Wu B, et al. Autophagy inhibition promotes paclitaxel-induced apoptosis in cancer cells. Cancer Lett. 2011;307(2):141-148.

19. Krek A, Grün D, Poy MN, et al. Combinatorial microRNA target predictions. Nat Genet. 2005;37(5):495-500.

20. Livak KJ, Schmittgen TD. Analysis of relative gene expression data using real-time quantitative PCR and the 2(-Delta Delta C(T)) Method. Methods. 2001;25(4):402-408.

21. Schmittgen TD, Livak KJ. Analyzing real-time PCR data by the comparative C(T) method. Nat Protoc. 2008;3(6):1101-1108.

22. Kyrgiou M, Salanti G, Pavlidis N, Paraskevaidis E, Ioannidis JP. Survival benefits with diverse chemotherapy regimens for ovarian cancer: meta-analysis of multiple treatments. J Natl Cancer Inst. 2006;98(22):1655-1663.

23. Ambros V. MicroRNA pathways in flies and worms: growth, death, fat, stress, and timing. Cell. 2003;113(6):673-676.

24. O'Connell RM, Rao DS, Baltimore D. microRNA regulation of inflammatory responses. Annu Rev Immunol. 2012;30:295-312.

25. Yeh YM, Chuang CM, Chao KC, Wang LH. MicroRNA-138 suppresses ovarian cancer cell invasion and metastasis by targeting SOX4 and HIF-1 $\alpha$. Int J Cancer. 2013;133(4):867-878.

26. Xu CX, Xu M, Tan L, et al. MicroRNA miR-214 regulates ovarian cancer cell stemness by targeting p53/Nanog. J Biol Chem. 2012;287(42): 34970-34978.

27. Qin W, Ren Q, Liu T, Huang Y, Wang J. MicroRNA-155 is a novel suppressor of ovarian cancer-initiating cells that targets CLDN1. FEBS Lett. 2013;587(9):1434-1439.

28. Noguer-Dance M, Abu-Amero S, Al-Khtib M, et al. The primatespecific microRNA gene cluster (C19MC) is imprinted in the placenta. Hum Mol Genet. 2010;19(18):3566-3582.

29. Asselin E, Mills GB, Tsang BK. XIAP regulates Akt activity and caspase-3-dependent cleavage during cisplatin-induced apoptosis in human ovarian epithelial cancer cells. Cancer Res. 2001;61(5):1862-1868.

30. Cheng JQ, Jiang X, Fraser M, et al. Role of X-linked inhibitor of apoptosis protein in chemoresistance in ovarian cancer: possible involvement of the phosphoinositide-3 kinase/Akt pathway. Drug Resist Updat. 2002;5(3-4):131-146.

31. Sasaki H, Sheng Y, Kotsuji F, Tsang BK. Down-regulation of X-linked inhibitor of apoptosis protein induces apoptosis in chemoresistant human ovarian cancer cells. Cancer Res. 2000;60(20):5659-5666.

32. Ma JJ, Chen BL, Xin XY. XIAP gene downregulation by small interfering RNA inhibits proliferation, induces apoptosis, and reverses the cisplatin resistance of ovarian carcinoma. Eur J Obstet Gynecol Reprod Biol. 2009;146(2):222-226.

33. Siegel C, Li J, Liu F, Benashski SE, McCullough LD. miR-23a regulation of X-linked inhibitor of apoptosis (XIAP) contributes to sex differences in the response to cerebral ischemia. Proc Natl Acad Sci U S A. 2011;108(28):11662-11667.

34. Xie Y, Tobin LA, Camps J, et al. MicroRNA-24 regulates XIAP to reduce the apoptosis threshold in cancer cells. Oncogene. 2013;32(19): 2442-2451.

35. Niederer F, Trenkmann M, Ospelt C, et al. Down-regulation of microRNA-34a* in rheumatoid arthritis synovial fibroblasts promotes apoptosis resistance. Arthritis Rheum. 2012;64(6):1771-1779. 


\section{Publish your work in this journal}

OncoTargets and Therapy is an international, peer-reviewed, open access journal focusing on the pathological basis of all cancers, potential targets for therapy and treatment protocols employed to improve the management of cancer patients. The journal also focuses on the impact of management programs and new therapeutic agents and protocols on
Dovepress

patient perspectives such as quality of life, adherence and satisfaction. The manuscript management system is completely online and includes a very quick and fair peer-review system, which is all easy to use. Visit http://www.dovepress.com/testimonials.php to read real quotes from published authors.

\footnotetext{
Submit your manuscript here: http://www.dovepress.com/oncotargets-and-therapy-journal
} 